\title{
Theoretical approaches for studying anisotropic negative thermal expansion: A case of cordierite
}

\author{
Takero TOKIZONO, ${ }^{\dagger, \dagger}$ Yusuke TSURU, ${ }^{*}$ Teruo ATSUMI,,$*$ \\ Norikazu HOSOKAWA ${ }^{* * *, \sharp}$ and Toshiharu OHNUMA ${ }^{* * *, *}$ \\ TOTO LTD., 2-8-1 Honson, Chigasaki, Kanagawa 253-8577, Japan \\ *TOTO LTD., 2-1-1 Nakashima, Kokura-kita-ku, Kitakyushu 802-8601, Japan \\ **TAIYO YUDEN CO., LTD., 5607-2 Nakamuroda-machi, Takasaki, Gunma 370-3347, Japan \\ ${ }^{* * *}$ DENSO CORPORATION, 500-1 Minamiyama, Komenoki-cho, Nisshin, Aichi 470-0111, Japan \\ ${ }^{* * * *}$ Central Research Institute of Electric Power Industry, 2-11-1 Iwado Kita, Komae, Tokyo 201-8511, Japan
}

\begin{abstract}
First-principles approaches on reproduction of negative and anisotropic thermal expansion of ceramics were examined. We have tested quasi-harmonic free-energy calculation and molecular dynamics (MD) simulation including lattice change based on the density functional theory. For intuitive understanding, a classical force-field MD was also applied. As a test case, we have chosen cordierite, which is particularly important for recent application for electronic precision components, catalytic converter for automobile exhaust gas, and is known to show negative and anisotropic thermal expansion at a certain region of temperatures. Quasi-harmonic free-energy approach showed appearance of negative expansion of cordierite in certain temperatures, while the anisotropy was not reproduced. We conclude this may be due to lack of anisotropic lattice change throughout the optimization of the unit cell and internal coordinates under hydrostatic pressures at $0 \mathrm{~K}$. On the other hand, MD simulation allowing lattice change showed anisotropy of $a$-, $b$-axes and $c$-axis of cordierite while the negative expansion region in temperature was not found, which may be due to limitation of available size of unit cell. Furthermore, by performing classical force-field MD, we found that local chemical-bond nature is a key in understanding the behavior of the cordierite under finite temperatures. From current simulated results, we propose necessity of intensive works to compare theoretical work with future accessible experiments using single-crystal samples.
\end{abstract}

@2016 The Ceramic Society of Japan. All rights reserved.

Key-words : Anisotropic thermal expansion, First-principles calculation, Quasi-harmonic approximation, Free energy, Molecular dynamics simulation, Cordierite

[Received October 1, 2015; Accepted April 2, 2016]

\section{Introduction}

First-principles simulation is a powerful tool to understand electronic structure and thermal properties of solids. Applications of the density functional theory (DFT) to compute free-energy for thermal expansion of solid states were demonstrated. In order to reproduce the thermal expansion, precise calculation of freeenergy of solid state under finite temperature is necessary. The practical ways of free-energy calculations are 1) calculating entropy by phonon to compute Helmholtz's free-energy ${ }^{1)}$ and 2) performing ab-initio molecular dynamics (MD) simulation under constant-temperature and pressure allowing change of lattice vectors. ${ }^{2), 3)}$ We have examined these methods for applicability in studying cordierite which possesses negative and anisotropic thermal expansion coefficient. For an intuitive understanding, MD simulation with classical force field ${ }^{4), 5)}$ was also performed.

Cordierite $\left(\mathrm{Mg}_{2} \mathrm{Al}_{4} \mathrm{Si}_{5} \mathrm{O}_{18}\right)$ is ceramics with extremely low thermal expansion coefficient in anisotropic axes of single crystalline phase. A good thermal shock resistance due to its low thermal expansion makes cordierite being one of attracting industrial materials which are applied as catalytic converter of auto-

Corresponding author: T. Tokizono; E-mail: takero.tokizono@jp. toto.com

* Physics session meeting in Computer aided Materials and Molecular design (CAMM) forum, 5-7-2 Kojimachi, Chiyoda$\mathrm{ku}$, Tokyo 102-0083, Japan mobile exhaust gas, the ceramic heater, and the structural member for the semiconductor exposure apparatus. Despite advances in experimental research, ${ }^{6), 7)}$ report by simulations that verifies the low thermal expansion has not yet been made.

In this letter, we demonstrate presence of certain temperature region that shows negative thermal expansion of cordierite as well as anisotropic thermal expansion by performing the DFT calculations for the free-energy within quasi-harmonic approximation, and for MD simulation under constant temperature and pressure. Thanks to the MD simulation based on the classical force-field model, we reproduced current results and deduced that the origin of the anisotropic low thermal expansion comes from local atomic coordinates around $\mathrm{Mg}$ atoms. ${ }^{8), 9)}$

\section{Computational method}

2.1 The Helmholtz free-energy derived from the first-principles phonon calculation

Within the quasi-harmonic approximation, the Helmholtz freeenergy at finite temperature is given by ${ }^{1)}$

$$
E=U+\frac{1}{2} \sum_{q} \hbar \omega_{q}+k_{B} T \sum_{q} \ln \left[1-\exp \left(-\frac{\hbar \omega_{q}}{k_{B} T}\right)\right],
$$

where $U$ is the total energy at absolute zero, $q$ is the wave vector, $\omega$ is the phonon frequency, $T$ is the temperature. $k_{B}$ and $\hbar$ are the Boltzmann constant and the reduced Planck constant, respectively.

For the first-principles calculation, we employed the plane- 


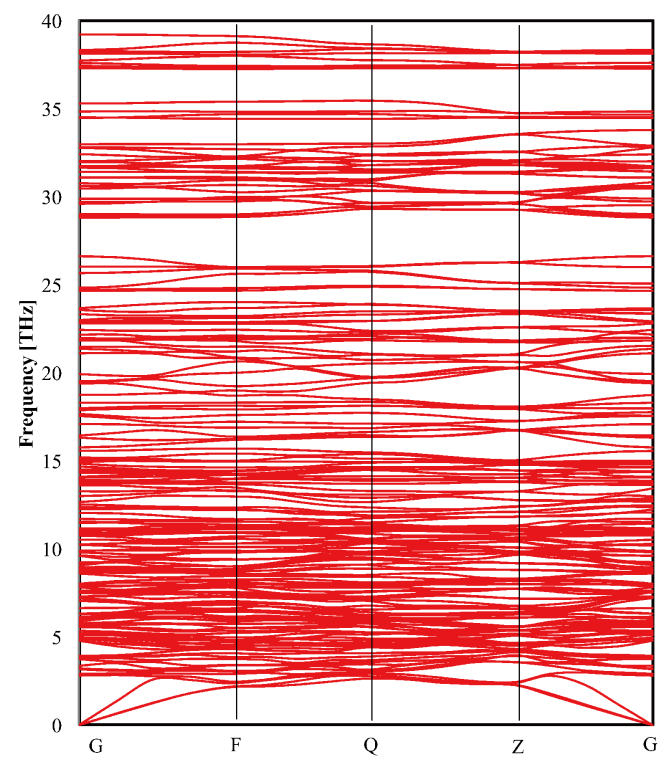

Fig. 1. The phonon band structure of the cordierite.

wave band-structure scheme with use of pseudopotentials ${ }^{10)}$ in the framework of the density functional theory ${ }^{11), 12)}$ as implemented in the CASTEP code. ${ }^{13)}$ The local density approximation (LDA) in CA-PZ (Ceperley and Alder ${ }^{14)}$ as parameterized by Perdew and Zunger ${ }^{15)}$ ) was used for exchange-correlation functional. Norm-conserving pseudopotentials ${ }^{16), 17)}$ were employed with the maximum cutoff energy of the plane wave basis set of $800 \mathrm{eV}$. The Brillouin zones of the unit cells were sampled by a $2 \times 2 \times 2$ k-point mesh. Electronic energy minimization was performed with a self-consistent field tolerance of $5.0 \times 10^{-7}$ $\mathrm{eV} /$ atom. The total energy $\mathrm{U}$ at absolute zero and the zero point energy [second term of Eq. (1)] were calculated with primitive cell model of orthorhombic cordierite (space group 66, Cccm, 58 atoms) and converted to $P 1$.

Phonon calculations were performed by the linear response method. ${ }^{18)}$ Helmholtz free energies $\mathrm{F}$ were calculated with $3 \times$ $3 \times 3$ q-vector mesh and $0.1 \AA^{-1}$ q-vector grid spacing. In Fig. 1, the phonon band structure of cordierite is shown. There is no soft mode in the phonon band structure. In Fig. 2, total energies at finite temperature with each volume at 0 to $1000 \mathrm{~K}$ are shown. To vary the volume, hydrostatic pressure was applied to the model. Solid state equation of Birch-Murnaghan ${ }^{19), 20)}$ was used to determine the minimum energy at each temperature and that of volume. By plotting the relationship between the temperature and volume obtained here, we obtained the thermal expansion curve.

\subsection{First-principles MD simulation}

For the first-principles MD simulation, we employed the planewave basis pseudopotential approach using CASTEP code, and the PAW (Projector Augmented Wave method) method using VASP code ${ }^{21), 22)}$ in the framework of the density functional theory as implemented. The MD simulations were performed twice with different initial conditions under each temperature of $0,200,400,600,800,1000,1200^{\circ} \mathrm{C}$.

In using the CASTEP code, Parrinello-Rahman NPT ensemble was employed ${ }^{2), 3)}$ being combined with Nose-Hoover thermostat. ${ }^{23)}$ An external pressure was set to $0 \mathrm{GPa}$. The local density approximation in CA-PZ was used for exchange-correlation functional. Ultrasoft pseudopotentials ${ }^{24)}$ were employed with the maximum cutoff energy of the plane wave basis set of $380 \mathrm{eV}$.

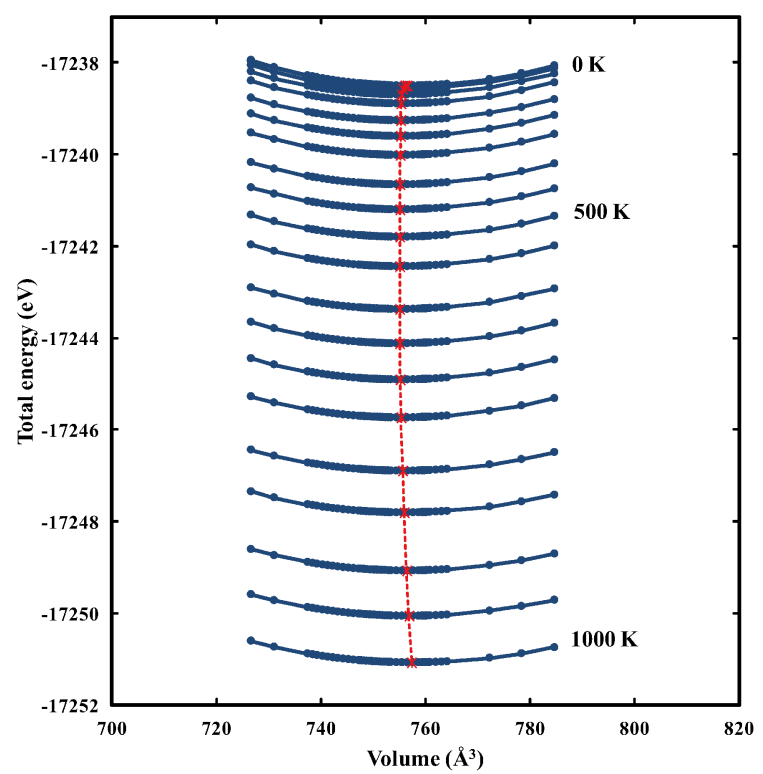

Fig. 2. Correlation of the Helmholtz free-energy with the volume of the cordierite at 0 to $1000 \mathrm{~K}$ using first-principles phonon analysis. The solid curves show the fitting curve. The minimum values of the fitting curves at temperatures are depicted by the crosses. The dashed curve passing through the crosses is guide to the eye.

The Brillouin zones of the unit cells were sampled by a $2 \times 2 \times 2$ k-point mesh. The supercell model of orthorhombic cordierite (space group 66, Cccm, 116 atoms) which was converted to $P 1$ was used. For the MD simulations, the interval of the time step $2 \mathrm{fs}$ and the total time period of $1 \mathrm{ps}$ (500 steps) were taken. For the values of lengths of $a, b$, and $c$ axes and cell volume, we took a time-average from 0.4 to $0.9 \mathrm{ps}$.

The robustness in the results of the MD simulation was confirmed by using the VASP code. In the calculations using VASP, the numerical schemes are different from those employed in using the CASTEP code. Parrinello-Rahman NPT ensemble was employed being combined with Langevin thermostat. ${ }^{25)}$ An external pressure was set to $0 \mathrm{GPa}$. The generalized gradient approximation (GGA) ${ }^{26}$ ) of Perdew-Burke-Ernzerhof (PBE) was used for exchange-correlation functional. ${ }^{27)}$ A plane-wave energy cutoff of $500 \mathrm{eV}$ was used. The Brillouin zones of the unit cells were sampled by a $\Gamma$ point. The supercell model of orthorhombic cordierite (space group 66, Cccm, 116 atoms), which was converted to $P 1$, was used. For the MD simulations, the interval of the time step $2 \mathrm{fs}$ and the total time period of $60 \mathrm{ps}$ $(30,000$ steps) were taken. For the values of lengths of $a, b$, and $c$ axes and cell volume, we took a time-average from 2 to $60 \mathrm{ps}$.

\subsection{Classical MD simulation}

For the classical MD simulation, we employed Berendsen NPT ensemble $^{28)}$ in the LAMMPS. ${ }^{29)}$ The $4 \times 4 \times 4$ supercell model of orthorhombic cordierite $\left(\mathrm{Mg}_{512} \mathrm{Al}_{1024} \mathrm{Si}_{1280} \mathrm{O}_{4608}\right)$ was used. The potentials of the Miyake ${ }^{30)}$ was employed. The potential equation is given by

$$
\begin{aligned}
\phi_{i j}\left(r_{i j}\right)= & \frac{z_{i} z_{j} e^{2}}{r_{i j}}+f_{0}\left(B_{i}+B_{j}\right) \exp \left(\frac{A_{i}+A_{j}-r_{i j}}{B_{i}+B_{j}}\right) \\
& -\frac{C_{i} C_{j}}{r_{i j}^{6}}+D_{i j}\left\{\operatorname { e x p } \left[-2 \xi_{i j}\left(r_{i j}-r_{i j}^{*}\right)\right.\right. \\
& \left.\left.-2 \exp \left[-\xi_{i j}\left(r_{i j}-r_{i j}^{*}\right)\right]\right]\right\},
\end{aligned}
$$

where $r$ is the distance between two atoms, $e$ is the electronic 
Table 1. Parameters of Miyake potential

\begin{tabular}{ccccccccc}
\hline Ion & $z$ & $a$ & $b$ & $c$ & Ion & $D$ & $\xi$ & $r^{*}$ \\
& $\mathrm{e}$ & $\AA$ & $\AA$ & $\mathrm{kJ}^{1 / 2} \AA^{3} / \mathrm{mol}^{1 / 2}$ & pair & $\mathrm{kJ} / \mathrm{mol}$ & $\AA^{-1}$ & $\AA$ \\
\hline $\mathrm{O}$ & -0.96 & 1.7700 & 0.138 & 51.23 & & & & \\
$\mathrm{Si}$ & 1.92 & 0.5983 & 0.025 & 0.00 & $\mathrm{Si}-\mathrm{O}$ & 63.0 & 2.0 & 1.47 \\
$\mathrm{Al}$ & 1.44 & 0.6758 & 0.030 & 0.00 & $\mathrm{Al}-\mathrm{O}$ & 50.4 & 2.0 & 1.58 \\
$\mathrm{Mg}$ & 0.96 & 0.9400 & 0.040 & 20.49 & $\mathrm{Mg}-\mathrm{O}$ & 42.0 & 2.0 & 1.75 \\
$\mathrm{Ca}$ & 0.96 & 1.1425 & 0.042 & 30.74 & $\mathrm{Ca}-\mathrm{O}$ & 21.0 & 2.0 & 2.20 \\
\hline
\end{tabular}

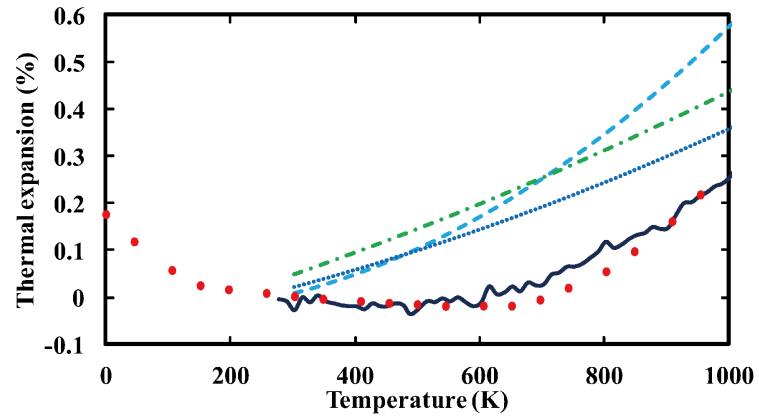

Fig. 3. Volume thermal expansion curve of cordierite calculated by first-principles phonon analysis (circle), experimental results from Fischer et al. (dashed curve), ${ }^{32)}$ Predecki et al. (dashed-dotted curve), ${ }^{7)}$ Lee et al. (dotted curve), ${ }^{33)}$ and Ikawa et al. (solid curve). ${ }^{6}$

charge, $f_{0}\left[=6.9511 \times 10^{-11} \mathrm{~N}=4.1861 \mathrm{~kJ} /(\mathrm{mol} \cdot \AA)\right]$ is the constant. $A, B, C$, and $z$ are parameters of each atom, $D, \xi$, and $r^{*}$ are parameters of each pair of cation-anion. These parameters are shown in Table 1. The cutoff distance of $12 \AA$ was used. The interval of the time step $1 \mathrm{fs}$ and the total time period of $6.15 \mathrm{~ns}$ $(6,150,000$ steps $)$ were taken. To make the initial structure, the supercell model was equilibrated at $300 \mathrm{~K}$ in the first $50 \mathrm{ps}$ and cooled to $50 \mathrm{~K}$ in the next $100 \mathrm{ps}$. The temperature was elevated $50 \mathrm{~K}$ every $200 \mathrm{ps}$ from 50 to $1500 \mathrm{~K}$. The first $100 \mathrm{ps}$ was used for equilibration and a time-average of the last $100 \mathrm{ps}$ was used for the values of lengths of $a, b$, and $c$ axes and cell volume.

\section{Results and discussion}

\subsection{Negative volume expansion by quasi harmon- ic approach}

Volume thermal expansion curves of cordierite are shown in Fig. 3. Volume thermal expansion of cordierite is defined by $(1 / V) \cdot \mathrm{d} V / \mathrm{d} T=\alpha_{a}+\alpha_{b}+\alpha_{c}$, where $\alpha_{a}, \alpha_{b}, \alpha_{c}$ are the linear expansion coefficient of each axis. Differences between the experimental works are probably due to the limit of experimental precision in measuring the small thermal expansion of cordierite or by due to the fact that samples were not single crystals as assumed in current simulations. In view of this dispersion, our calculation was generally consistent with the experiments.

The linear expansion curve is shown in Fig. 4. The thermal expansion anisotropy ( $a, b$-axes are positive, but $c$ axis is negative) was seen in the experiments, but not in the calculation. The reason for this was considered as following. The anisotropy did not appear in the structure optimization applied hydrostatic pressure in the $0 \mathrm{~K}$. This anisotropy might be obtained if we searched the most stable axial length independently changing the $a-, b$-, and $c$-axes respectively, like Ogata et al. ${ }^{31)}$ To reproduce the anisotropy, we tried the first-principles MD simulation as presented in the next subsection.
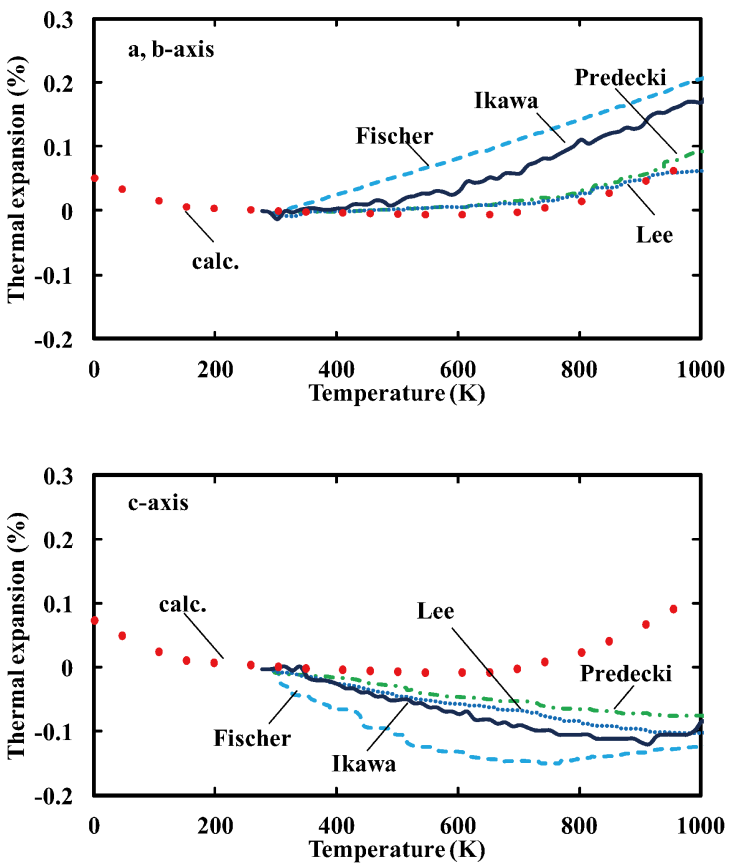

Fig. 4. Thermal expansion of $a, b$-axes and $c$-axis. Circle are results of current simulation. While experimental results were denoted by allow with authors names, Fischer et al. (dashed curve), ${ }^{32)}$ Predecki et al. (dashed-dotted curve), ${ }^{7}$ Lee et al. (dotted curve), ${ }^{33)}$ and Ikawa et al. (solid curve). ${ }^{6}$

Table 2. The target temperature and the average temperature at the firstprinciples molecular dynamics simulation by using CASTEP and VASP

\begin{tabular}{cccccccc}
\hline Target temp. $\left[{ }^{\circ} \mathrm{C}\right]$ & 0 & 200 & 400 & 600 & 800 & 1000 & 1200 \\
\hline $\begin{array}{c}\text { Average temp. } \\
\text { by using } \\
\begin{array}{c}\text { CASTEP }\left[{ }^{\circ} \mathrm{C}\right] \\
\text { Average temp. }\end{array} \\
\begin{array}{c}\text { by using } \\
\text { VASP }\left[{ }^{\circ} \mathrm{C}\right]\end{array}\end{array}$ & -3.7 & 194.1 & 393.2 & 589.8 & 795.4 & 986.1 & 1187.4 \\
\hline
\end{tabular}

\subsection{Constant pressure MD simulation showing anisotropy}

To consider anisotropy, the first-principles MD simulation of constant pressure was performed. The first-principles MD was performed by using CASTEP and VASP codes. CASTEP code, which was used for the phonon calculation, was again used to confirm that the first-principles MD simulation was able to reproduce the anisotropy. VASP code was used to perform the long scale $(60 \mathrm{ps})$ calculation which was necessary to add credibility. The reason to use VASP code for long-time MD simulations is the authors' computational resources and we believe there should no essential difference in results obtained by these codes except for details originating from difference in numerical conditions. Table 2 shows the target temperature and the average temperature in the first-principles MD simulation. The target temperature from 0 to $1000^{\circ} \mathrm{C}$ was well controlled, because the difference between the target temperature and the average temperature was several degrees. At $1200^{\circ} \mathrm{C}$, the average temperatures were lower $12.6^{\circ} \mathrm{C}$ (CASTEP) or $16.7^{\circ} \mathrm{C}$ (VASP) than the target, but they were sufficiently controlled because the errors were only about $0.9 \%$ (CASTEP) or $1.1 \%$ (VASP) at the absolute temperature. 
Figure 5 shows the temperature dependence of the volume thermal expansion obtained by the first-principles MD simulation. Unlike the phonon calculation, the volume increased against temperature monotonically. Figure 6 shows the temperature dependence of the length of $a-, b-, c$-axes. Besides detailed difference between results by CASTEP and VASP due to different time-constant for the MD-simulation, both codes derived a common trend; The $a$ - and $b$-axes increased monotonically with temperature, but the $c$-axis was found to exhibit negative. (VASP code shows minimum of thermal expansion of $c$-axis at $600^{\circ} \mathrm{C}$.) This is the reproduction of the tendency of the anisotropy of the thermal expansion of cordierite in the experiment. On the other hand, unlike experiments, the volume expansion increased

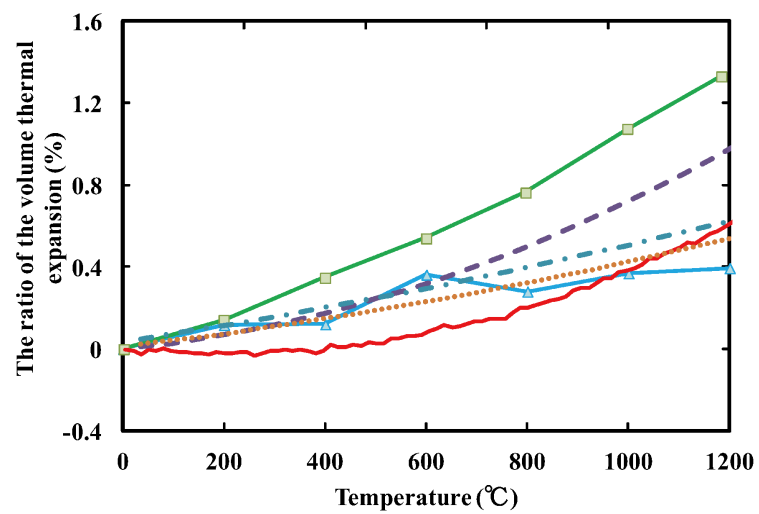

Fig. 5. Correlation of the temperature with the ratio of the volume thermal expansion of the cordierite using first-principles molecular dynamics simulation: Experimental result from Fischer et al. (dashed curve), Predecki et al. (dashed-dotted curve), Lee et al. (dotted curve), and Ikawa et al. (solid curve), calculated by using CASTEP ( $\boldsymbol{\Delta}$ ), by using VASP ( $\square)$.

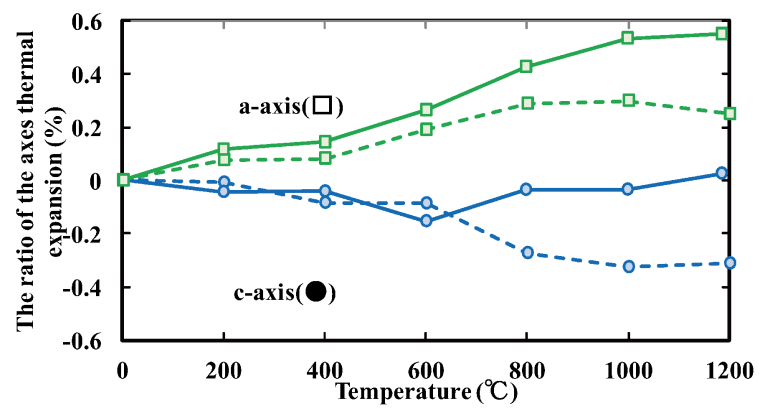

Fig. 6. Correlation of the temperature with the axis ratio of the cordierite using first-principles molecular dynamics by using CASTEP (dashed curve) and VASP (solid curve). The axis ratio was calculated by the axis length at each temperature divided by the length at $0^{\circ} \mathrm{C}$. monotonic. This is probably because of limited numerical precision in describing small proportion of the negative expansion of $c$-axis.

The correlation of the bond length and axis thermal expansion was investigated. Table 3 shows $\mathrm{Al}-\mathrm{O}, \mathrm{Mg}-\mathrm{O}, \mathrm{Si}-\mathrm{O}$ bond lengths and $a, b, c$-axes lengths of the 0 and $600^{\circ} \mathrm{C}$ and the difference between them. These were obtained by performing the structural optimization at $0 \mathrm{~K}$ for the location of the atom at 0 and $600^{\circ} \mathrm{C}$. The different binding distances of the $\mathrm{Mg}-\mathrm{O}$ and $\mathrm{Al}-\mathrm{O}$ mean the different binding ways. The bond lengths with temperature of $\mathrm{Al}-\mathrm{O}$ and $\mathrm{Si}-\mathrm{O}$ were small, which were $0.1 \%$ or less, but that of $\mathrm{Mg}-\mathrm{O}$ was about 0.2 to $0.5 \%$. The change of $a$, $b$-axes were about $0.4 \%$, so the thermal expansion of $a, b$-axes were caused by a change in bond length of $\mathrm{Mg}-\mathrm{O}$, and these results suggested that the anisotropic thermal expansion was caused by different temperature-dependence of each bond.

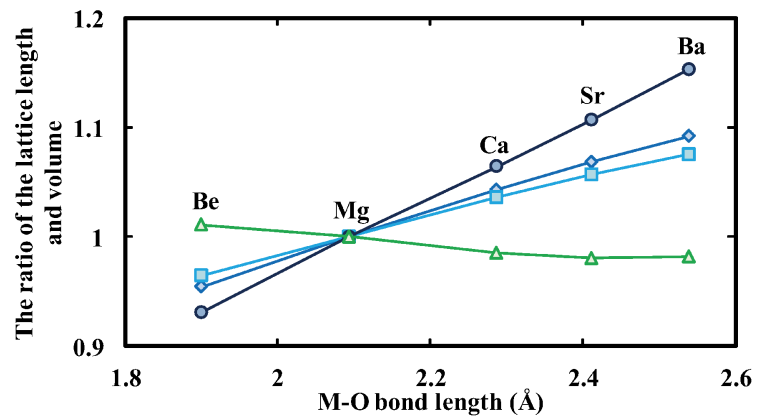

Fig. 7. Correlation of the $\mathrm{M}-\mathrm{O}$ distance with the ratio of the lattice length ( $a$-axis: $\boldsymbol{\nabla}, b$-axis: $\boldsymbol{\square}, c$-axis: $\mathbf{\Delta}$ ) and the volume:

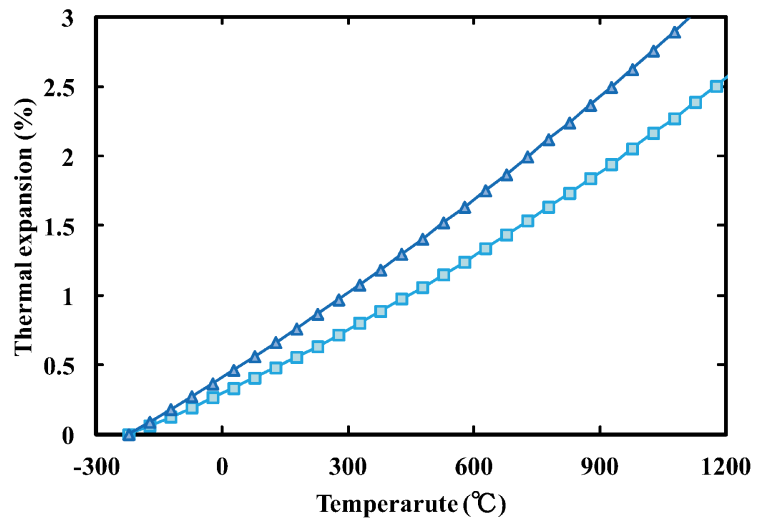

Fig. 8. Correlation of the temperature with volume thermal expansion of the $\mathrm{Mg}$-cordierite and $\mathrm{Ca}$-cordierite by using classical MD (Mgcordierite: $\boldsymbol{\Delta}$, Ca-cordierite: $\square$ ).

Table 3. The correlation of the axes length with the bond lengths of $\mathrm{Mg}-\mathrm{O}, \mathrm{Al}-\mathrm{O}$, and $\mathrm{Si}-\mathrm{O}$ at the temperature of 0 and $600{ }^{\circ} \mathrm{C}$

\begin{tabular}{cccccccccc}
\hline CASTEP & $\mathrm{Mg}-\mathrm{O}$ & $\mathrm{Mg}-\mathrm{O}$ & $\mathrm{Mg}-\mathrm{O}$ & $\mathrm{Al}-\mathrm{O}$ & $\mathrm{Al}-\mathrm{O}$ & $\mathrm{Si}-\mathrm{O}$ & $a$-axis & $b$-axis & $c$-axis \\
\hline Bond length at $0^{\circ} \mathrm{C}[\AA]$ & 2.0895 & 2.0963 & 2.0979 & 1.7053 & 1.7362 & 1.6004 & 16.9210 & 9.6197 & 9.2233 \\
Bond length at $600^{\circ} \mathrm{C}[\AA]$ & 2.0939 & 2.1018 & 2.1036 & 1.7068 & 1.7380 & 1.6017 & 16.9475 & 9.6479 & 9.2153 \\
Difference & $0.21 \%$ & $0.27 \%$ & $0.27 \%$ & $0.09 \%$ & $0.10 \%$ & $0.08 \%$ & $0.16 \%$ & $0.29 \%$ & $-0.09 \%$ \\
\hline & & & & & & & & \\
\hline VASP & $\mathrm{Mg}-\mathrm{O}$ & $\mathrm{Mg}-\mathrm{O}$ & $\mathrm{Mg}-\mathrm{O}$ & $\mathrm{Al}-\mathrm{O}$ & $\mathrm{Al}-\mathrm{O}$ & $\mathrm{Si}-\mathrm{O}$ & $a$-axis & $b$-axis & $c$-axis \\
\hline Bond length at $0^{\circ} \mathrm{C}[\AA]$ & 2.1246 & 2.1298 & 2.1360 & 1.7724 & 1.7731 & 1.6478 & 17.2665 & 9.8353 & 9.4473 \\
Bond length at $600^{\circ} \mathrm{C}[\AA]$ & 2.1307 & 2.1384 & 2.1466 & 1.7733 & 1.7743 & 1.6498 & 17.3121 & 9.8794 & 9.4328 \\
Difference & $0.29 \%$ & $0.40 \%$ & $0.50 \%$ & $0.05 \%$ & $0.07 \%$ & $0.12 \%$ & $0.26 \%$ & $0.45 \%$ & $-0.15 \%$ \\
\hline
\end{tabular}



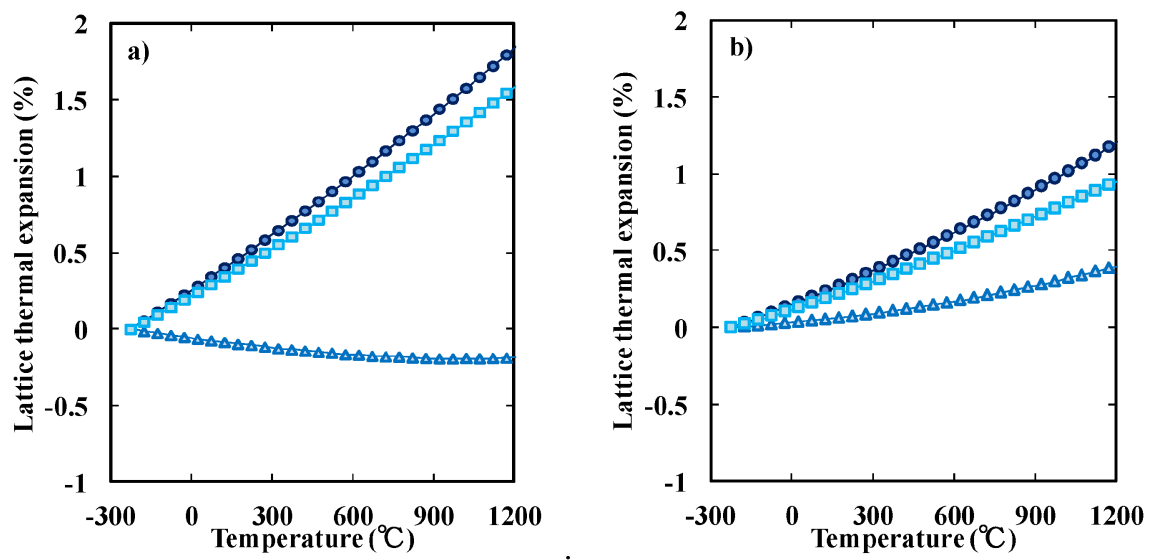

Fig. 9. Correlation of the temperature with lattice thermal expansion of the a) $\mathrm{Mg}$-cordierite and b) Ca-cordierite by using classical MD ( $a$-axis:,$b$-axis: $\square, c$-axis: $\mathbf{\Delta}$ ).

By using CASTEP, the effect of the substitution $\mathrm{Mg}$ with other alkali earth metal $(\mathrm{Be}, \mathrm{Ca}, \mathrm{Sr}, \mathrm{Ba})$ on the thermal expansion was investigated. When the $\mathrm{M}-\mathrm{O}$ distance increased, $a, b$-axes extended and $c$-axis shrank, but the volume increased (See Fig. 7). Similar tendency was obtained in GGA. Thus, it was revealed $\mathrm{Mg}-\mathrm{O}$ bond length was affecting the thermal expansion anisotropy.

\subsection{Classical MD simulation for Mg substitution}

In performing classical MD simulation, the effect of substituting $\mathrm{Mg}$ with $\mathrm{Ca}$ on the anisotropy was investigated. Figures 8 and 9 show temperature dependence of the volume and the lattice constant of $\mathrm{Mg}$-cordierite and Ca-cordierite respectively. The simulated change of the lattice constants could reproduce the anisotropy agreeing with the results by first-principles MD simulations. The volume thermal expansions of both cordierites increased monotonically with temperature, and these were overestimated the experimental result. However, the anisotropy of $\mathrm{Ca}$-cordierite was less than $\mathrm{Mg}$-cordierite. This suggests the anisotropy is affected to the existence of $\mathrm{Mg}$ in the cordierite. In order to examine the dependence of the inter-atomic potentials, we also tested the Matsui's potential, ${ }^{34)}$ and obtained the same tendency.

\section{Conclusions}

We have demonstrated usefulness of the computational approaches to simulate anisotropic and negative thermal expansion of cordierite. By performing the first-principles phonon calculation to derive the Helmholtz free-energy, the negative thermal expansion at a certain temperature region was reproduced. Firstprinciples MD simulations allowing lattice motion could reproduce anisotropy in positive and negative thermal expansions appeared in $a, b$-axes and $c$-axis, respectively, which was attributed as the temperature dependence of the $\mathrm{Mg}-\mathrm{O}$ bond lengths. Moreover, classical force-field MD performed by checking sizeeffect of the unit cell also could reproduce anisotropic thermal expansion, which was found to be suppressed by substituting an $\mathrm{Mg}$ atom to a $\mathrm{Ca}$ atom. This finding suggests the role of $\mathrm{Mg}$ atoms in triggering the anisotropy in thermal expansion. We conclude that reproduction of experimental results by combination of several computational schemes for materials with anisotropic thermal expansion having complex structures like as cordierite is promising. Further intensive comparison, for example, between measured data of the single crystal and corresponding theoretical computations is expected to derive guiding principles for designing novel materials that satisfies industrial requirements.

Acknowledgement We would like to express our special appreciation Dr. Yoshiyuki Miyamoto from Advanced Industrial Science and Technology (AIST) giving us much advises. We also would like to thank Computer aided Materials and Molecular design (CAMM) forum providing us with an opportunity to discuss and write this thesis. We acknowledge for the forum membership, Dr. Shin'ichi Higai from Murata Manufacturing Co., LTD., Dr. Masahiro Tanaka from Kyocera Corp., Dr. Yoshiki Iwazaki from TAIYO YUDEN Co., LTD., Dr. Takehiro Suzuki from Noritake Co., Limited, Mr. Hideyuki Omura from Canon Inc., Dr. Kiyoshi Betsuyaku from Central Research Institute of Electric Power Industry (CRIEPI), Dr. Tokutaro Komatsu from Kyoto University, Ms. Karin Furukawa from Hitachi Zosen Co., Dr. Kengo Nishio from AIST, Mr. Atsushi Fujiwara from Ryoka Systems Inc., and Ms. Reiko Usui, in charge of CAMM forum.

\section{References}

1) A. Togo, L. Chaput, I. Tanaka and G. Hug, Phys. Rev. B, 81, 174301 (2010).

2) M. Parrinello and A. Rahman, Phys. Rev. Lett., 45, 1196-1198 (1980).

3) A. Rahman and N. Parrinello, J. Appl. Phys., 52, 7182-7190 (1981).

4) P. S. Baram and S. C. Parker, Philos. Mag. B, 73, 49-58 (1996).

5) M. J. Sanders, M. J. Leslie and C. R. A. Catlow, J. Chem. Soc. Chem. Commun., 1271-1273 (1984).

6) H. Ikawa, T. Otagiri, O. Imai, M. Suzuki, K. Urabe and S. Udagawa, J. Am. Ceram. Soc., 69, 492-498 (1986).

7) P. Predecki, J. Haas, J. Faber, Jr. and R. L. Hitterman, J. Am. Ceram. Soc., 70, 175-182 (1987).

8) J. P. Cohen, F. K. Ross and G. V. Gibbs, Am. Miniral, 62, 6778 (1977).

9) M. F. Hochella, Jr., G. E. Brown, Jr., F. K. Ross and G. V. Gibbs, Am. Miniral, 64, 337-351 (1979).

10) P. Hohenberg and W. Kohn, Phys. Rev., 136, B864-870 (1964).

11) W. Kohn and L. J. Sham, Phys. Rev., 140, A1133-1138 (1965).

12) G. B. Bachelet, D. R. Hamann and M. Schlüter, Phys. Rev. B, 26, 4199-4228 (1982).

13) M. C. Payne, M. P. Teter, D. C. Allan, T. A. Arias and J. D. Joannopoulos, Rev. Mod. Phys., 64, 1045-1098 (1992).

14) D. M. Ceperley and B. J. Alder, Phys. Rev. Lett., 45, 566-568 (1980).

15) J. P. Perdew and A. Zunger, Phys. Rev. B, 23, 5048-5079 (1981). 
16) J. S. Lin, A. Qteish, M. C. Payne and V. Heine, Phys. Rev. B, 47, 4174-4180 (1993).

17) M. H. Lee, PhD Thesis, Cambridge University (1996).

18) S. Baroni, S. D. Gironcoli, A. D. Corso and P. Giannozzi, Rev. Mod. Phys., 73, 515-564 (2001).

19) F. Birch, Phys. Rev., 71, 809-824 (1947).

20) F. D. Murnaghan, Proc. Natl. Acad. Sci. U.S.A., 30, 244-247 (1944).

21) P. E. Blochl, Phys. Rev. B, 50, 17953 (1994).

22) G. Kresse and D. Joubert, Phys. Rev. B, 59, 1758-1775 (1999).

23) W. G. Hoover, Phys. Rev. A, 31, 1695-1697 (1985).

24) D. Vanderbilt, Phys. Rev. B, 41, $7892-7895$ (1990).

25) M. P. Allen and D. J. Tildesley, Computer simulation of liquids, Oxford university press: New York (1991).

26) J. P. Perdew, K. Burke and M. Ernzerhof, Phys. Rev. Lett., 77,
3865-3868 (1996).

27) J. P. Perdew, K. Burke and Y. Wang, Phys. Rev. B, 54, 1653316539 (1996).

28) H. J. C. Berendsen, J. P. M. Postma, W. F. van Gunsteren, A. DiNola and J. R. Haak, J. Chem. Phys., 81, 3684-3690 (1984).

29) S. J. Plimpton, J. Comput. Phys., 117, 1-19 (1995).

30) A. Miyake, Mineral. J., 20, 4, 189-194 (1998).

31) K. Matsubara, H. Kimizuka and S. Ogata, J. Soc. Mater. Sci., Jpn., 63, 188-193 (2014).

32) G. R. Fischer, D. L. Evans, and J. E. Geiger, Abstract B-18. p. 214 in Crystalloaraphic Association Program and Abstracts Series 2, Vol. 2 (1974).

33) J. D. Lee and J. L. Pentecost, J. Am. Ceram. Soc., 59, 183 (1976).

34) M. Matsui, Mineral. Mag., 58A, 571-572 (1994). 